\title{
Agent Based Framework Architecture for Supporting Content Adaptation for Mobile Government
}

\author{
http://dx.doi.org/10.3991/ijim.v7i1.2131 \\ Hasan Omar Al-Sakran, Qasem Kharma, Irina Serguievskaia \\ King Saud University, Saudi Arabia
}

\begin{abstract}
Rapid spread of smart mobile technology that supports internet access is transforming the way governments provide services to their citizens. Mobile devices have different capabilities based on the manufacturers and models. This paper proposes a new framework for adapting the content of M-government services using mobile agent technology. The framework is based on a mediation architecture that uses multiple mobile agents and XML as semistructure mediation language. The flexibility of the mediation and XML provide an adaptive environment to stream data based on the capabilities of the device sending the query to the system.
\end{abstract}

Index Terms-Agent, Mobile-government, Content Adaptation, E-government, mediation.

\section{INTRODUCTION}

Due to the latest advances in wireless mobile communication infrastructure and web technologies citizens, businesses, and public agencies are using wireless technologies in order to do their daily transactions.

One of the important transactions categories is governmental transactions such as submitting and following up applications, querying records, etc. These data are distributed over many ministries' systems that may use different software applications and apply different restrictions on accessing data. In other words, the data sources are heterogeneous in the structure and naming conventions. On the other hand, mobile technologies or what is called mobility nowadays offers many services and applications that enhance our lifestyle. The technology is fast growing and the number of users is increasing dramatically as well. In the past few years we have observed a rapid evolution of wireless technologies and a widespread of internetenabled mobile devices. In the mid of 2005 , the number of cellular phones per person was over three times higher than PCs, and most of sophisticated phones now have the processing power of a mid-1990s PC, and it is increasing [1]. Moreover, mobile phones are a relatively low-cost technology so practically everyone can afford it, and their usage is relatively simple comparing to Internet technology [4]. In 2006 about one billion people, worldwide, purchased a new handset [2]. The number of mobile users is increasing rapidly and in 2010 , according to an international statistics, there were 5.3 billion mobile subscriptions, which means $77 \%$ of world population [3]. There are rapid advances in mobile technologies and services.

Mobile devices can be categorized into main three categories as following:
- Personal Digital Assistant (PDA): PDAs are small handheld devices which have some of the personal computers capabilities as well as telephone capabilities. PDAs offer many interesting functionalities such as: organizing personal schedules, multimedia support, recognizing text and voice input. They offer the user the ability to connect the Internet to check an email or to search the web [5].

- Cellular Phones: In the last few years, cellular phones spread all over the world. Cellular phones range from devices with limited functionalities that are used for voice and short text message communications to advanced devices, like third generation phones $(3 \mathrm{G})$, which allow the user to connect to the Internet, to send or check e-mails and to open small web pages. For that, the flexibility of these devices is less than the flexibility of PDAs [5].

- Smartphone: Smartphone is hybrid devices that take some abilities from PDAs and other abilities from cellular phones. Smartphone can be used for text and voice communication, e-mail, web access and as media or video player.

Mobile devices have different operating systems and different capabilities such as different connection speed, different resolutions, etc. The major challenges of wireless network are low bandwidth, low quality connections, and the limitations of portable devices such as screen size, power consumption, processing, and memory.

The continuous improvement and development of mobile and wireless technologies provides new opportunities and challenges for mobile e-government. Using mobile technologies benefit the users in different ways. One of the benefits is the accessibility of the government services from anywhere and anytime. Use of wireless and mobile devices allows governments to provide citizens with efficient public services and to share information within and between agencies [6], [7]. To stay relevant, governments should take action and use the opportunities to enhance the usability and accessibility of their services through mobile devices. Without a doubt, mobile government is one of the most recent technology trends that use mobile phones to access government websites and services. The advancements in wireless technologies play the major role in growing of the number of worldwide mobile Internet subscribers who have a wireless access to various online recourses through mobile devices. Such facts reveal the importance of moving from E-government to M- 
PAPER

government and they can be considered as the driving forces toward the adoption of M-government [8].

In this paper we propose a framework solving the aforementioned problems: accessing governmental heterogeneous data sources and manipulating different presentations for mobile devices. The solution is based on a mediation framework that is based on collaboration of mobile and stationary agents. These agents are used to get data from different ministries and capable of solving the problem of having different data structures and naming conventions. When a new device connects to the mediator, the device will promote its capabilities that will be taken into consideration by specific agent which will prepare the returning data with corresponding style sheet supporting the mobile device. Therefore, the framework is capable of handling heterogeneous data source and delivering data in different presentation.

The rest of the paper is organized as follows. Section 2 briefly reviews related work for mediation for content adaptation for mobile E-government. Section 3 presents an overview of M-government. Section 4 presents the details of the proposed architecture including agent technology. Section 5 concludes the paper and suggests direction for future work.

\section{RELATED WORK}

Significant amount of research have been conducted to deal with content adaptation issues on mobile devices. Different approaches of content adaptation have been proposed to resolve these issues. Fudzee and Abawajy [9] classified these approaches into static adaptation which generates and stores multiple variants of the content and dynamic adaptation where the adaptation of the content is done on fly during the user's request based on his mobile device specifications. Adaptation can be performed at the client side, at the intermediary proxy server, or at the server side where the content is stored [10]. Shahidi et al. [11] proposed a method where a large number of adaptation mechanisms conducted at different locations in the network.

The advantage of the client-side adaptation is that users with more powerful devices can define preferences and determine the type and scale of the adaptation process. But not all devices are capable to deal with highcomplexity applications, and the adaptation software should be generated for different classes of devices. With the server side approach the content at every web page can be adapted for specific needs of the requesting mobile device. The transformed content is fully controlled by the content provider. Unfortunately the content provider should maintain and update database of all client mobile device types and has to implement all possible content adaptations. The intermediary proxy server approach can be implemented regardless of the content providers and can be used for different contents types by applying set of general transformation rules. It is an efficient solution for mobile government and its implementation is relatively easy.

Different mediation frameworks have been proposed for designing the content of M-government services to resolve heterogeneous data sources integration and content adaptation. A number of authors suggested to customize Web content according to the mobile client contextual environments, including personal preferences, access condition, network connection, and device capabilities $[12,13,14,15,16]$, while others proposed frameworks that capable of recognizing mobile devices' technologies, understanding their implications for M-government applications, and adapting of the content on mobile devices $[17,18]$. J. He et al. applied a rule based approach to guide content adaptations [19].

Dickson et al. [20] proposed a framework of three tiers of views: user interface view, data view, and process view. The process view serves as a mechanism for integrating user interface view and data view. Sheng [5] discussed some technical and policy considerations related to M-government. One of the important issues that were described in this paper is the content and presentation management. The authors suggested some useful guidelines to handle this issue exploiting techniques such as: utilizing Content Management Systems (CMS) to add a formal structure to the content and to adopt enterprisewide web and content design standards, using the Extensible Markup Language (XML), Extensible Style sheet Language (XSL), and employing Simple Object Access Protocol (SOAP) technology.

Beside M-government issues and challenges, Sharma and Gupta [21] presented a web services architecture, while Abramowicz et al. discussed the design issues of user interface and the requirements of mobile user representations [22]. Amailef and Lu [23] present a framework of mobile government in emergency cases. The framework supports interactions between government, citizens, and other non-government agencies in emergency situations. Similar methods for content adaptation to support mobile devices have been suggested in [24, 25] where intermediary services adapt the content provided by a server to the context of the user.

Authors' interests lie in different research direction: implementing an agent technology to solve the content adaptation problem. Minjie LI et al. [26] use agents for content adaptation and solving the problem of temporary disconnection in wireless mobile network applications. Hongtao $\mathrm{Zhu}$ and Fangli Su [27] suggested an Egovernment integration model based on the semantic web, web services and intelligent agent technologies.

\section{MOBILE GOVERNMENT:}

Governments have created new channels of government services delivery known as Mobile Government (Mgovernment) aimed at engaging citizens and improving system efficiency [25].Mobile government (Mgovernment) extends E-government benefits from its minimal requirement which is a personal computer (PC) to mobile devices by implementing wireless technologies. M-government is considered a supplementary approach of delivery of governmental services from distributed heterogeneous data sources using different transmission channels and technologies [28]. M-government is defined by Kushchu \& Kuscu as "a strategy and its implementation involving the utilization of all wireless and mobile technologies, services, applications and devices for improving benefits to the parties involved in e-government including citizens, businesses and all government units" [29]. In this work we define M-government as the flexible provider of public services through mobile and wireless technologies to support users anytime and anywhere. 
PAPER

The M-government practices can be classified into informational, transactional and operational functions [30]. Informational functions are one way transmission of information from government to a user. These functions provide governmental information to citizens via publishing and broadcasting. It can also send alerts and notifications to a user through SMS or e-mails. Transactional functions are two way transmissions of information from government to a user and vice versa. This class of functions allows a user to interact with the M-government system, such as online procurement and payments. Operational functions aim to handle the internal governmental operations. It enables government employees to access some important information from remote locations through their mobile devices. In this work we are focusing on the content of the informational and operational functions and how this content must be personalized and adapted to meet the user preferences and technologies.

Saldhana [31] and Kumar et al. [32] stated that there are four different types of interaction levels where $M$ government operates represented as follow:

- MGovernment-to-government $(\mathrm{mG} 2 \mathrm{G})$ that is interactions between government agencies;

- MGovernment-to-employee (mG2E) that is interactions between government and its employees;

- MGovernment-to-citizen $(\mathrm{mG} 2 \mathrm{C})$ that is interactions between government and citizens; and

- MGovernment-to-business (mG2B) that is interactions between government and businesses.

The government services provided through mobile devices are called mobile services (m-service). This domain of M-government encompasses very critical services that would benefit citizens. The criteria of evaluating the mobile government services are: websites usability, SMS notifications and extra m-services. Main categories of usability issues are screen design, content, accessibility and navigation. The category of SMS notifications includes sending messages to and receiving notifications from ministries. Any services designed for mobile phone users not included in two first categories represent the category of extra m-services for example downloadable maps, m-books, m-brochures, etc.

L. Knutsen, et al. categorized mobile services as follows. First category is represented by the mobile content and information services (e.g. map, location-based services, news, and entertainment content downloads). Another category is messaging services such as short message service (SMS), multimedia messaging service (MMS) and email service. Transaction-based services such as mobile banking and airline reservations are yet another category of m-services [33].

Kuscu [34] and Su and Pei [35] identified many useful M-government applications that have been used. These applications include SMS for people with hearing disabilities, flood and fire notifications, search for missing children, automobile parking, tax declaration, mobile elderly care workers, garbage collection, reporting crime, etc.

In spite of all benefits and advantages discussed so far, implementing M-government is complex. Interoperability (variety of platforms, etc.) and usability (mobile devices limitations) are some of the challenges that the implementers of M-government are facing. Success of mobile government will depend largely on the number of its users. That's why implementation of user-friendly and easy access to information in alternative forms is a must.

\section{M-GOVERnMENT AgENT-BASEd FramewORK}

As we have mentioned previously, M-government informational functions can provide end users with published online information and can send alerts and notifications to users while operational functions enable government employees to access any needed information from remote locations. In the following sections, we introduce our adaptive M-government framework based on mediation architecture and highlight some useful guidelines that must be applied in order to design information content, which is provided by the M-government platform, displayed efficiently and effectively.

\section{A. Agent technology:}

Agent is intelligent computational reusable executable software on a network, which can carry out autonomous tasks or delegate the execution of these tasks to another agent or set of agents. It has a degree of control over its actions and does not always have to wait for commands. It adapts its behavior in response to the changing environment. A mobile agent has the property that its state and code can be moved to another server within the same network, and then continues executing from where it stopped on the previous server. The operation of agents is supported and managed by distributed agent platforms systems, an agent can migrate from server to server in heterogeneous environments. On each server an agent interacts with stationary services and other resources to accomplish its mission. It can communicate to anticipate, adopt and plan tasks. Its behavior consists of beliefs, desires, and interaction depending on the location of an entity within an agent-based system. Agent technology represents excellent solutions for solving problems in large-scale distributed systems, in our case for the egovernment applications. E-government system by nature is distributed, and the integration of data from distributed ministries' servers takes a long time. Mobile agents can migrate to ministries' servers and process data locally, thus significantly reducing the response time. Such a system supports intelligent and distributed storage, allows an optimal personalized m-government environment, supports an intelligent decision-making, and ensures interoperability between different systems that are to be integrated into an operational heterogeneous e-government system. Besides that, it solves the problem of heterogeneity and low-bandwidth, reduces network traffic, process data locally instead of transmitting the data over a network and supports disconnected operation. Using agent components could accelerate development; enhance modularity, reusability, flexibility and reliability. When a mobile agent is dispatched there is no need to wait for it to return. The original connection to the network does not need to remain maintained while the mobile agents are out. The mobile agents can wait until the original connection is reestablished before attempting to return.

\section{B. System overview}

The architecture of proposed m-government agentbased system has a 3-tier structure involving the client mobile devices, the web server holding the mediator, and the ministry application servers as shown in Figure1. 
PAPER

Mobile Client

Mediator Web Application

Ministry Site

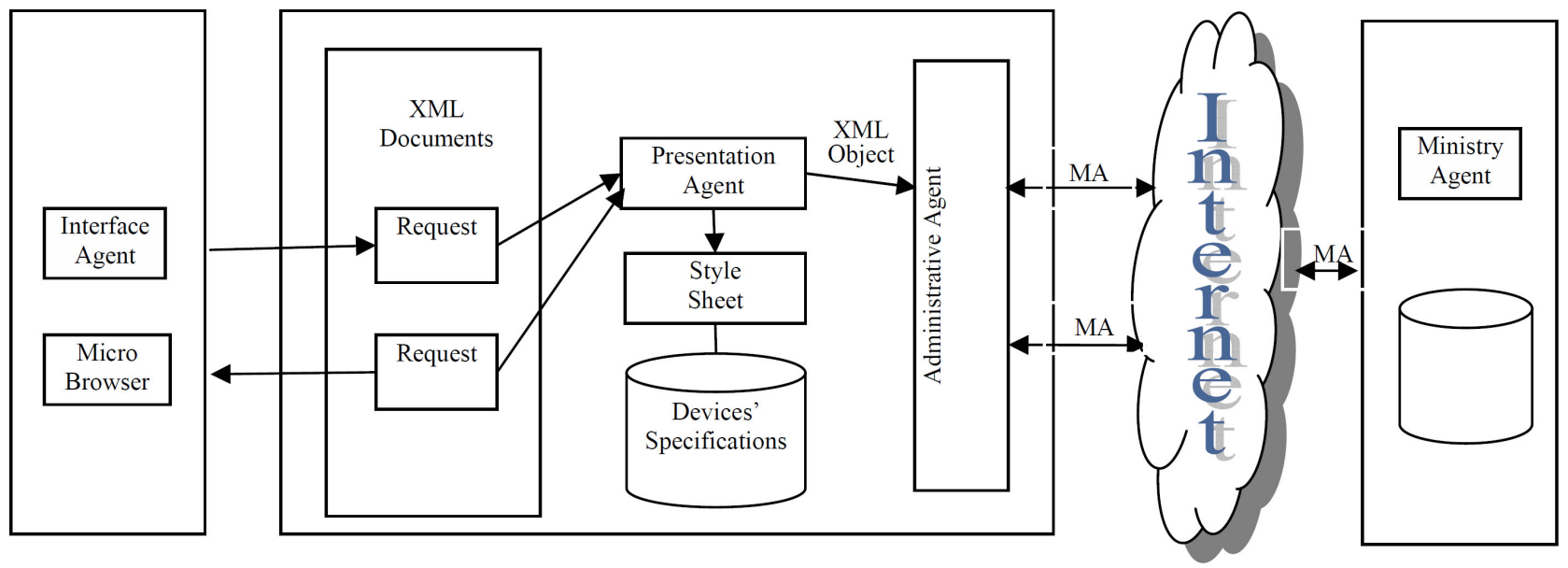

Figure 1. System Architecture

The main components of the architecture and their functionalities can be summarized as follows:

1. Client who accesses the Internet using a wireless Internet device via a micro browser. Mobile browsing is based on the Wireless Application Protocol (WAP). WAP is a protocol that was designed to allow the users to browse the Internet from their mobile device. This protocol can view the information that is written by Wireless Markup Language (WML). Pages need to be reformatted to narrow and tall view to fit small displays. This reformatting technique may affect the order of presenting the content. Client fills the request form and the browser submits it. The platform receives the requests of the client sends the request to the web server, and save the request in a temporary mobile agent.

2. Intermediate adaptation web server: This tier responsible for content adaptation. It is a key part in the process of designing M-government applications with regard to the adaptation of the content to meet the user preferences and the different capabilities and limitations of mobile devices and wireless technologies that are used by different users. This server is an intermediary server which placed between a mobile client and an application server, and it controls the adaptation process. It is in charge of content delivery. The server holds stationary agents (administrating and presentation agents), user profile, and device specification database. These stationary agents execute only on the web server where it begins execution, and their code cannot be changed during execution. The user profile is created when the user requests service(s) for the first time. It contains the client's device specification that provides the services requested by the user and the list of services based on user's preferences. The agent specifies which presentation type is most appropriate to the user according to predefined set of rules. As a result, each user can receive an adaptive content that meet his preferences and is compatible with his mobile device and wireless technology.

3. Ministry Application server: Ministries' sites provide the services to satisfy the requests from the incoming mobile agents through their ministry agents. By nature data sources are distributed and heterogeneous, and their structures are different. In order to manipulate those sources efficiently, data must be homogenized to resolve these differences. All the data will be converted into an XML object before being passed to the mobile agents.

\section{Dedicated agents:}

Agents need a running agent platform (agent execution environment) on every server they might move to (on mobile device, mediator, and ministries application servers) to send or receive mobile agents. Several mobile agent platforms have been proposed to include java agents such as IBM'Aglets, ObjectSpace's Voyager, Mitsubishi's Concordia, and General Magic's Odyssey. The combination of agent platform environments and the micro edition of Java (J2ME) technology provide a good solution for building very sophisticated mobile applications on most of mobile devices.

1. Interface Agent: it provides user-friendly interactive interface for mobile user. It is a static agent within the client mobile device.

2. Mobile agents: Mobile agent can move from one system to another. Mobile agents can be generated dynamically during the execution. It can reconfigure itself dynamically based on changes of the services.

3. Administrating agent: The administrating agent will generate and send one or multi mobile agents to the appropriate ministries sites. This agent is responsible for the connectivity to ministries' Databases, thus eliminating a connection per request. When mobile agents are back, the administrating agent will analyze the collected information based on client preference.

4. Presentation agent: With the emergence of heterogeneous devices, adaptation became unavoidable. It's main goal to enable the presentation of e-government content of on different mobile devices. The device context is information that is used to characterize the user mobile device. It is crucial to specify the user device's capabilities in M-government application because they can have a big impact on what content is appropriate. Some of the parameters that characterize mobile devices are the main factors that will be used to characterize this context, such as device type and device screen resolution. Nowadays mobile de- 
vices can be connected to Internet through different wireless technologies. Each of them has different data transfer rate. As a result, we have to specify the type of wireless technology that will be used by the user to connect his device to the Internet; this is called the connectivity context. The agent takes responsibility for detecting the mobile device capabilities (memory, screen size and resolution), mobile browser, and information about users, such as location, time, and network performance using user profile and device specification databases. Based on these features the agent will create adaptive content for specific characteristics of mobile device. The presentation agent will convert the result to the XSL or other style according to the wireless device. Style adaptation is achieved with device-dependent style sheets (in the form of CSS, XSL or XSLT). CSSstyle used to remove background shading and increase font sizes [36].

5. Ministry agent: receives the request from mobile agent and searches the local ministry's database. Initially the result is presented in XML document, and then converted into an XML object. The XML object is passed to the mobile agent who can go back to the web server and pass the result to administrating agent of the mediator. Then presentation agent converts the XML object into XML document again.

In case of reliable connection, the mobile client request is sent to the mediator and saved there. The mediator creates an agent or multiple agents to carry the request to the e-government application servers where it is processed and the result is send back by the agent(s). The presentation agent represents the result in the XSL or other style according to the requesting device's specifications and then delivers it back to the mobile device.

Agent technology is an effective approach to handle weak connectivity and disconnection problems in wireless networks (whether a mobile client sends to or receives data from the e-government servers). In such cases, the mediator saves the result in a temporary mobile agent. When the connection is re-established that agent migrates to the mobile device with the result. Then the browser of the mobile device displays the result. If the client device is offline at the time, the mediator will send email or SMS to the client. In case of no wireless connection, the interface agent saves the request of the client and as soon as the connection is re-established the interface agent via a mobile agent passes the request to the mediator where it will be processed in the same way as mentioned above.

\section{CONCLUSIONS}

The work presents a new framework for m-government system based on agent technology that improves the provision of M-government services by delivering personalized and adapted service content to the appropriate user efficiently and effectively. The proposed framework provides the ability to access e-government services over mobile devices which allow citizens to expand the capabilities of their devices. The agent approach described in this framework offers enough flexibility through a collection of dedicated agents. The proposed framework used a mediation architecture that can handle heterogeneous data sources, and adapt different presentation to the same data based on the characteristics of the connected client's device. The future work will be the implementation of this framework using Aglets platform with the micro edition of Java (J2ME).

\section{REFERENCES}

[1] Georgiev, T., Georgieva, E., \& Trajovski, G.: "Transitioning from e-Learning to m-Learning: Present issues and future challenges", Proc. of the Seventh ACIS International Conference on Software Engineering, Artificial Intelligence, Networking, and Parallel/ Distributed Computing (SNPD '06), (2006).

[2] Misuraca, G.: " Exploring the potential of Mobile Internet Services in emerging countries: a look towards the future", Proc. of 12th EADI General Conference- Global Governance for Sustainable Development: The Need for Policy Coherence and New Partnerships, Geneve, (2008).

[3] Telecompaper, mobile subscribers in 2010 in the world, www.telecompaper.com

[4] Mengistu, D. \& Zo, H. \& Rho, J. J. (2009), M-Government: Opportunities and Challenges to Deliver Mobile Government Services in Developing Countries, 2009 Fourth International Conference on Computer Sciences and Convergence Information Technology, p.1445-1450 http://dx.doi.org/10.1109/ICCIT.2009. 171

[5] Sheng, H.; Trimi, S.: "M-government: technologies, applications and challenges", Electronic Government, an International Journal, Vol. 5, No. 1, pp.1-18, (2008).

[6] Moon, M. J. (2004). From E-Government to M-Government? Emerging Practices in the Use of Mobile Technology by State Governments. Washington: IBM Center for The Business of Government.

[7] Khaled Amailef and Jie Lu, 2008,"m-Government: A Framework of Mobile-based Emergency Response Systems", Proceedings of 2008 3rd International Conference on Intelligent System and Knowledge Engineering

[8] Du Huiying, Lu Tingjie, Liu Jiale, Wu Miao,2010, “The consider on the construction of Mobile City combined with mobile computingtechnology: a case of mobile government", 2010 International Conference on Net working and Digital Society

[9] Fudzee, M.F.M.,Abawajy, J.:Aclassification for content adaptation system. In: Proceedings of iiWAS2008,pp. 426-429, Linz, Austria (2008)

[10] Velibor Adzic \& Hari Kalva \& Borko Furht "A survey of multimedia content adaptation for mobile devices", journal of Multimed Tools Appl (2011) 51:379-396

[11] Merat Shahidi, Nika Naghavi, A. Hamid Aghvami, Content Adaptor Selection Models in daptation Management Framework, 2011, 18th International conference on Telecommunications, IEEE, Cyprus May 2011. http://dx.doi.org/10.1109/CTS. $\underline{2011.5898971}$

[12] Stephen J.H. Yang, Jia Zhang, Angus F.M. Huang, Jeffrey J.P. Tsai, Philip S. Yu, "A Context-driven Content Adaptation Planner for Improving Mobile Internet Accessibility”, 2008 IEEE International Conference on Web Services.

[13] A. Pashtan, S. Kollipara, and M. Pearce. "Adapting Content for Wireless Web Service." IEEE Internet Computing, 7(5), 79-85, 2003. http://dx.doi.org/10.1109/MIC.2003.1232522

[14] S. Toivonen, J. Kolari, and T. Laakko. "Facilitating Mobile Users with Contextualized Content." Artificial Intelligence in Mobile System Workshop, Seattle, WA, USA, October, 2003.

[15] B. Kurz, I. Popescu, and S. Gallacher. "FACADE - A Framework for Context-Aware Content Adaptation and Delivery." Second Annual Conference on Communication Networks and Services Research, 46-55, 2004. http://dx.doi.org/10.1109/DNSR.2004. $\underline{1344710}$

[16] A. Krause, A. Smailagic, and D. P. Siewiorek. "Context-Aware Mobile Computing: Learning Context- Dependent Personal Preferences from A Wearable Sensor Array." IEEE Transactions on Mobile Computing, 5(2), Feb., 113-127, 2006. http://dx.doi.org/10.1109/TMC.2006.18

[17] Kumar, M.; Sinha, O.: "M-government - Mobile Technology for E-government ", Proc. of 5th international conference on egovernance (ICEG-2007), Hyderabad, India, (2007). 
[18] Guirguis, S.K. Hassan, M.A. 2010 "A smart framework for web content and resources adaptation in mobile devices", Advanced Communication Technology (ICACT), 2010 The 12th International Conference, 487 - 492 , Phoenix Park.

[19] Jiang He, Tong Gao, Wei Hao, I-Ling Yen, and Farokh Bastani, 2007, A Flexible Content Adaptation System Using a Rule-Based Approach, IEEE TRANSACTIONS ON KNOWLEDGE AND DATA ENGINEERING, VOL. 19, NO. 1, JANUARY 2007.

[20] Dickson K.W. Chiu, Dan Hong, S.C. Cheung, and Eleanna Kafeza, "Towards Ubiquitous Government Services through Adaptations with Context and Views in a Three-Tier Architecture", Proceedings of the 40th Hawaii International Conference on System Sciences - 2007

[21] Sushil K. Sharma and Jatinder N.D. Gupta. Web services architecture for m-government: issues and challenges. Electronic Government 1(4):462-474, 2004. $\quad$ http://dx.doi.org/10.1504 /EG.2004.005921

[22] Witold Abramowicz, Andrzej Bassara, Agata Filipowska, Marek Wiśniewski, Paweł Żebrowski. Mobile Implications for $\mathrm{m}$ Government Platform Design. Cybernetics \& Systems 27(23):119-135, 2006. http://dx.doi.org/10.1080/01969720500428255

[23] Khaled Amailef and Jie Lu "m-Government: A Framework of Mobile-based Emergency Response Systems" Proceedings of 2008 3rd International Conference on Intelligent System and Knowledge Engineering.

[24] D. Malandrino, F. Mazzoni, D. Riboni, C. Bettini, M. Colajanni, and V. Scarano, "MIMOSA: Context-aware adaptation for ubiquitous web access ". Personal \& Ubiquitous Copmut. 14(4), pp. 301-320, May 2010.

[25] Christos K. Georgiadis and Emmanouil Stiakakis,2010,"Extending an e-Government Service Measurement Framework to mGovernement Services, 2010 Ninth International Conference on Mobile Business / 2010 Ninth Global Mobility Roundtable.

[26] Minjie LI, Liqiang WANG and Ying HAO, "A Mobile Agents Based Framework in Wireless Network",The 2nd IEEE International conference on Information Management and engineering(ICIME),90-93, 2010.

[27] Hongtao Zhu and Fangli Su, "An Integrated Model in EGovernment Based on Semantic Web, Web Service and Intelligent Agent",2010, http://dl.ifip.org/index.php/ifip/article/view/10746 .
[28] Gasem

[29] Kushchu, I., \& Kuscu, H. (2003). From E-Government to Mgovernment: Facing the Inevitable, The 3rd European conference on E-Government (ECEG03), Trinity College, Dublin, p. 253-260

[30] Norris, D.F..; Moon, M.J.: "Advancing E-government at the grassroots: tortoise or hare?", Public Administration Review, Vol. 65, No. 1, pp.64-75, (2005). http://dx.doi.org/10.1111/j.15406210.2005.00431.x

[31] A. Saldhana. Secure E-Government Portals - Building a web of trust and convenience for global citizens. In W3C Workshop on eGovernment and the Web. 2007. Washington DC, USA.

[32] Kumar,M., Hanumanthappa, M. \& Reddy, B.L. "Security issues in m-government", Proc. of the International Conference on Global e-Security (ICGeS 2008), London, UK, CCIS 12, Springer-Verlag, 2008, pp. 265-273.

[33] L. Knutsen, et al., "Acceptance and perceptions of advanced mobile services: alterations during a field study," presented at the International Conference on Mobile Business, Sydney, Australia, 2005.

[34] Kuscu, M.; Kushchu, Ibrahim; Yu, Betty (2009), Mobile Computing,Introducing Mobile Government

[35] Chen Su and Zhou Pei 2010 "Application Model of Mobile EGovernment in Wuhan Urban Circle", 2010 International Conference on Multimedia Information Networking and Security http://dx.doi.org/10.1109/MINES.2010.157

[36] U. Erra, G. Iaccarino, D. Malandrino, and V. Scarano, "Personalizable edge services for web accessibility". Universal Access Inf. Soc. 6(3), pp. 285-306, 2007. http://dx.doi.org/10.1007/s10209-007-0091-y

\section{AUTHORS}

Hasan Omar Al-Sakran, Qasem Kharma, and Irina Serguievskaia are with King Saud University, Saudi Arabia.

Received 25 May 2012. Published as resubmitted by the authors 20 December 2012. 